\title{
Shikonin-induced Apoptosis of Colon Cancer Cells Is Reduced by Peroxiredoxin V Expression
}

\author{
NISANSALA CHANDIMALI ${ }^{1 *}$, HU-NAN SUN ${ }^{2 *}$, LING-ZU KONG ${ }^{1}$, \\ XING ZHEN ${ }^{1}$, REN LIU ${ }^{1}$, TAEHO KWON ${ }^{3}$ and DONG-SUN LEE ${ }^{1,4,5}$ \\ ${ }^{1}$ Interdisciplinary Graduate Program in Advanced Convergence Technology and Science, \\ Jeju National University, Jeju, Republic of Korea; \\ ${ }^{2}$ Department of Disease Model Animal Research Center, College of Life Science and Technology, \\ Heilongjiang Bayi Agricultural University, Daqing, P.R. China; \\ ${ }^{3}$ Primate Resources Center, Korea Research Institute of Bioscience and Biotechnology (KRIBB), \\ Jeongeup, Republic of Korea; \\ ${ }^{4}$ Subtropical/Tropical Organism Gene Bank, Jeju National University, Jeju, Republic of Korea; \\ ${ }^{5}$ Practical Translational Research Center, Jeju National University, Jeju, Republic of Korea
}

\begin{abstract}
Background/Aim: Colon cancer is the second most common deadliest malignancy in the world and better understanding of its underlying mechanisms is needed to improve clinical management. Natural plant extracts are gaining attention in the development of new therapeutic strategies against various cancer types. Shikonin is a naturally extracted naphthoquinone pigment with effects against cancer, including colon cancer. Materials and Methods: In this study, we conducted a series of in vitro experiments to show the effects of Shikonin on colon cancer cell apoptosis. A colon cancer cell line with overexpression of peroxiredoxin $V(\operatorname{PrxV})$ was constructed and the relationship of PrxV expression with Shikonin-induced cell apoptosis was investigated. Results: Shikonin induced colon cancer cell apoptosis via regulation of mammalian target of rapamycin signaling. Shikonin-induced cell apoptosis was abrogated by overexpression of PrxV. Conclusion: According to the results obtained in this study, targeting PrxV may provide new insight for the successful management of colon cancer by inducing cell apoptosis.
\end{abstract}

The second most common cause of cancer-related death worldwide, colon cancer, has been reported to be resistant to

\footnotetext{
*These Authors contributed equally to this study.

Correspondence to: Professor Dong-Sun Lee, Interdisciplinary Graduate Program in Advanced Convergence Technology \& Science, Jeju National University, Jeju 63243, Republic of Korea. Tel: +82 647543340, Fax: +82647513780, e-mail: dongsunlee@jejunu.ac.kr
}

Key Words: Peroxiredoxin V, colon cancer, mTOR, ROS, Shikonin. recommended therapies depending on the metastatic stage. Therefore, the urgent development of more efficient strategies remains a challenge for better management of colon cancer (1). Understanding the underlying mechanisms of colon cancer progression and drug resistance may provide new insights into successfully overcoming this challenge.

Compounds extracted from various plants are gaining global attention as successful therapeutic strategies for several types of cancer $(2,3)$. Shikonin, a natural naphthoquinone pigment compound purified from Lithospermum erythrorhizon (4), is one such natural compound which has been studied for its activities against various cancer types (5), including non-small cell lung cancer (4), nasopharyngeal carcinoma (6), and ovarian carcinoma (7). It has also already been reported that anticancer effects of Shikonin against colon cancer cells are mediated by inducing apoptotic cell death (8-11). Shikonin also has been identified as a regulator of several signaling pathways including phosphatase and tensin homolog (PTEN)/protein kinase B (AKT)/mammalian target of rapamycin (mTOR) signaling in endometrioid endometrial cancer cells (12), c-Jun N-terminal kinase (JNK), protein kinase transducer and activator of transcription 3 (STAT3)/protein kinase B (AKT) pathway in non-small cell lung cancer (13) and extracellular signal-regulated kinase (ERK)/JNK/mitogen-activated protein kinase (MAPK)/AKT pathway in leukemia cells (14).

Shikonin has also been reported to be a natural inducer of reactive oxygen species (ROS) in cancer cells leading to induction of apoptotic cell death (8). But the mechanism behind this induction in colon cancer cells has not yet been studied. Therefore, we focused on the antioxidant enzyme system of colon cancer cells in order to reveal the mechanism by which Shikonin regulates apoptotic cell death. 
Peroxiredoxins (Prxs) are an important superfamily of antioxidant enzymes and consists of six members (15). Among them, PrxV is elevated in colon cancer tissues compared with other Prx family members and normal colon tissues (16). Overexpression of PrxV was shown to inhibit apoptosis of colon cancer cells (16). But as far as we are aware, the association of $\operatorname{PrxV}$ expression and mTOR signaling has not yet been studied. Therefore, we mainly focused on the relationship between PrxV and Shikonininduced apoptosis via mTOR signaling pathway in colon cancer cells. We hypothesized that $\operatorname{PrxV}$ may play an important role in the regulation of colon cancer cell apoptosis induced by Shikonin.

\section{Materials and Methods}

Cell culture and generation of stable cell lines. HCT116, HT29, HT29 Mock, and Prx V-overexpressing HT29 cells (HT29 HisPrxV) cell lines were maintained in Dulbecco's modified Eagle's medium (Invitrogen, Carlsbad, CA, USA) supplemented with 10\% fetal bovine serum (FBS; Hyclone, Logan, UT, USA), penicillin $(100 \mathrm{U} / \mathrm{ml})$ and streptomycin $(100 \mathrm{mg} / \mathrm{ml})$ in an incubator at $37^{\circ} \mathrm{C}$ with $5 \%$ humidified $\mathrm{CO}_{2}$. HCT116 Mock, HCT116 shPrxV and HCT116 His-PrxV cell lines were established as described previously (17).

Apoptosis and proliferation assays. Cells were inoculated at a density of $5 \times 10^{3}$ cells/well in 96-well plates. Cell proliferation of HCT116, HT29, HT29 Mock and HT29 His-PrxV cells after 24-h treatment with Shikonin $(0,0.1,0.5,1,5,10,15$ and $20 \mu \mathrm{M})$ was studied using EZ-Cytox Kit (DoGenBio, Korea), according to the manufacturer's instructions. The absorbance was measured at 450 $\mathrm{nm}$. To detect apoptosis, cells were prepared using the propidium iodide (PI), Annexin V Detection Kit (BD Biosciences, CA, USA), according to the manufacturer's instructions, and analyzed by flow cytometry (FACSCalibur; BD Biosciences).

Western blot analysis. Treated HT29 and HCT116 cells were lysed in RIPA buffer (150 mM NaCl, $1 \%$ Nonidet p-40, $50 \mathrm{mM}$ Tris, $\mathrm{pH}$ 8.0 , and a protease inhibitor cocktail). Thirty micrograms of protein from each sample was separated by sodium dodecyl sulfate-PAGE and transferred to a nitrocellulose membrane (Bio-Rad, California, USA). Western blotting was performed with rabbit or mouse antibodies against PrxI, PrxII: LF-PA0091 (AbFrontier, Seoul, South Korea), PrxIII, PrxIV, PrxV, PrxVI, mTOR, pmTOR, AKT, pAKT, pERK, p38, pP38, and His-Prx V. Glyceraldehyde-3-phosphate dehydrogenase (GAPDH) was used as control. Protein expression levels were detected qualitatively using Super Signal West Pico PLUS Chemiluminescent Substrate (Thermo Fisher, CA, USA).

2',7'-Dichlorofluorescin diacetate (DCF-DA), dihydroethidium (DHE) and 5,5',6,6'-tetrachloro-1,1',3,3' -tetraethyl-benzimidazolylcarbocyanine chloride (JC-1) staining assays. Changes in the ROS levels of HT29 and HCT116 cells with dose-dependent treatment of Shikonin were determined using DCF-DA (Invitrogen). Cells were incubated with $20 \mathrm{mM}$ of DCF-DA, DHE and JC-1 respectively for $15 \mathrm{~min}$ at $37^{\circ} \mathrm{C}$. After washing with $1 \times \mathrm{PBS}$, images were taken under microscopy and the fluorescence intensities were noted qualitatively.
Transwell assays. Migration and invasion assays were performed using 24-well transwell chambers with $8.0-\mu \mathrm{M}$ pore polycarbonate membranes (Merck Millipore, Darmstadt, Germany) without (migration) or with (invasion) Matrigel. Briefly, 200- $\mu$ l volumes of HCT116 and HT29 cell suspensions in medium containing $0.5 \%$ FBS were added separately to the upper chambers $\left(1 \times 10^{5}\right.$ cells/chamber). In each case, the bottom chamber was filled with $800 \mu \mathrm{l}$ of medium supplemented with $20 \%$ FBS (or not) as a chemoattractant. The cells were then incubated for $24 \mathrm{~h}$ at $37^{\circ} \mathrm{C}$ in $5 \% \mathrm{CO}_{2}$. Cells that passed through the coated membrane to the lower surface were then fixed with $4 \%$ paraformaldehyde and stained with $0.1 \%$ crystal violet for $1 \mathrm{~h}$ prior to capturing images under a microscope. Images were captured and the mobilized cell numbers were counted in treated and non-treated groups comparatively.

Immunocytochemistry. HT29 cells treated with Shikonin in a dose-dependent manner were fixed with $3.7 \%$ formaldehyde in $1 \times$ PBS for $10 \mathrm{~min}$ at room temperature. Those cells were then blocked with $1 \mathrm{X}$ PBS containing $0.5 \%$ Triton $\times-100$ and $1 \%$ bovine serum for $60 \mathrm{~min}$ at room temperature and then incubated with primary antibodies against mTOR and pmTOR for $18 \mathrm{~h}$. On the following day, cells were washed with $1 \times$ PBS with Tween 20 and incubated with secondary antibodies for $2 \mathrm{~h}$ at room temperature. Nuclei were visualized by 4',6-diamidino-2phenylindole (DAPI) staining. Nuclear staining was observed qualitatively under a microscope after $20 \mathrm{~min}$ of DAPI staining, and images were acquired. Fluorescence intensities of images taken from treated and non-treated groups were examined comparatively.

Colony-formation assay. HT29 and HCT116 cells $\left(1 \times 10^{3}\right.$ cells/well) were plated in six-well plates and treated with $0,5,10$ and $20 \mu \mathrm{M}$ of Shikonin. Cells were maintained in an incubator at $37^{\circ} \mathrm{C}$ with $5 \% \mathrm{CO}_{2}$ for 7 days. Cells were then washed with $1 \times$ PBS, fixed with $3.7 \%$ formaldehyde for $10 \mathrm{~min}$, treated with methanol for $20 \mathrm{~min}$, and stained with crystal violet for $30 \mathrm{~min}$. The plates were washed three times with $1 \times$ PBS prior to image capturing. The number of colonies in treated and non-treated groups was counted.

Sphere-formation assay. HT29 and HCT116 cells $\left(2 \times 10^{3}\right.$ cells/well) were seeded in a six-well Ultra Low Cluster plate (Corning Inc., Corning, NY, USA) and cultured for 10 days in suspension in serum-free Dulbecco's modified Eagle's medium/F12 (Gibco, Grand Island, NY, USA) containing B27 supplement (1: 50; Invitrogen), 20ng/ml epidermal growth factor (Calbiochem, San Diego, CA, USA), and $0.5 \%$ bovine serum albumin (SigmaAldrich, St. Louis, MO USA). The spheres were imaged with an inverted microscope and counted. Sphere-formation efficiency was calculated as the number of colonies/input cells $\times 100 \%$. Immunocytochemical staining of spheres was performed following the steps of the assay as described above in the section on immunocytochemistry.

Statistical analysis. The data are depicted as the mean \pm SEM. Student's $t$-tests were performed using GraphPad Prism 4.0 software (GraphPad Software Inc., San Diego, CA, USA), and values of $* p<0.05$ were considered indicative of significant difference. 

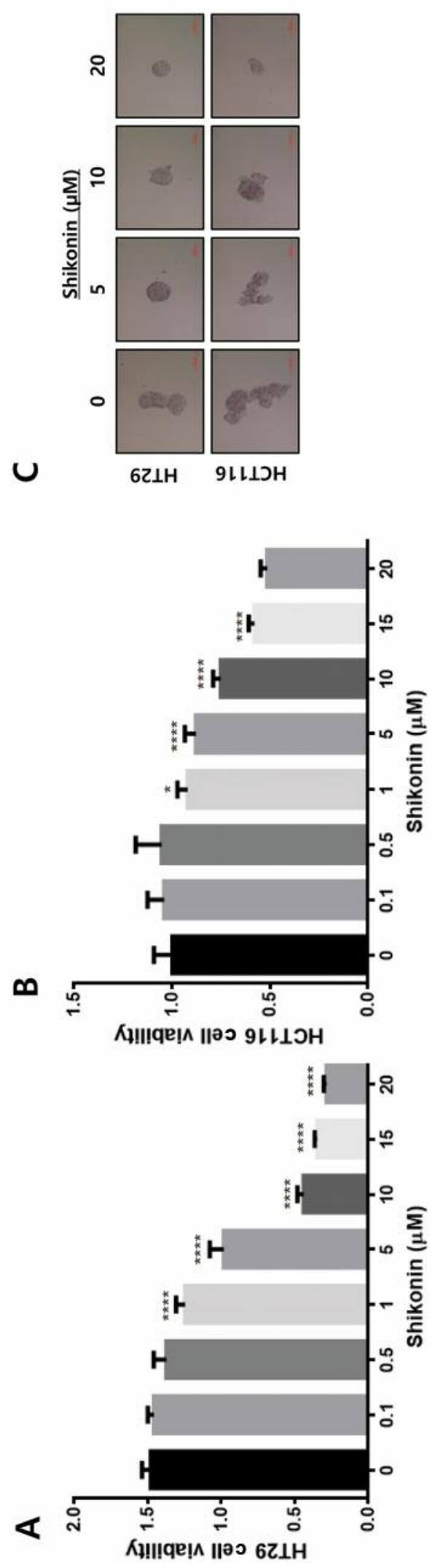
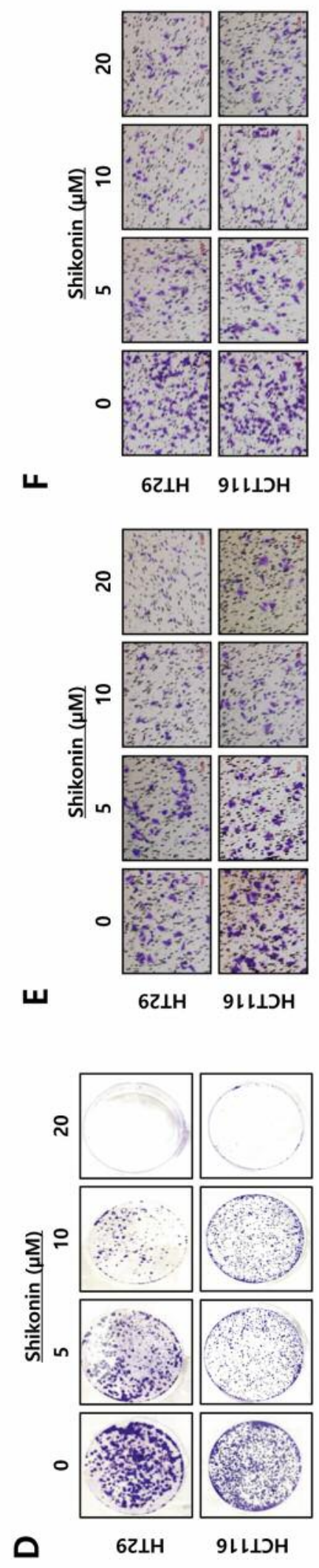

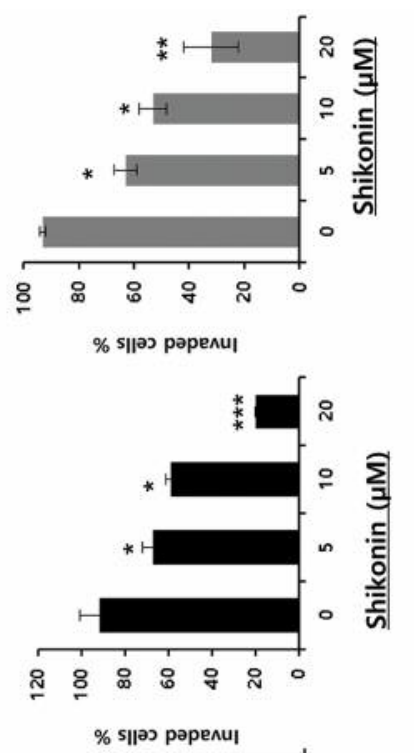

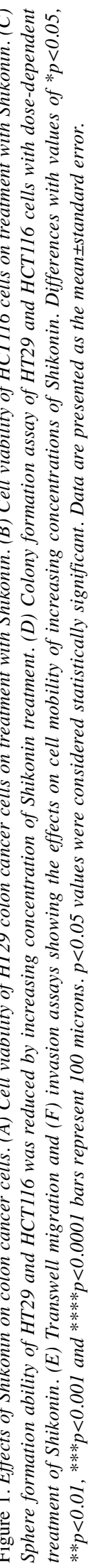




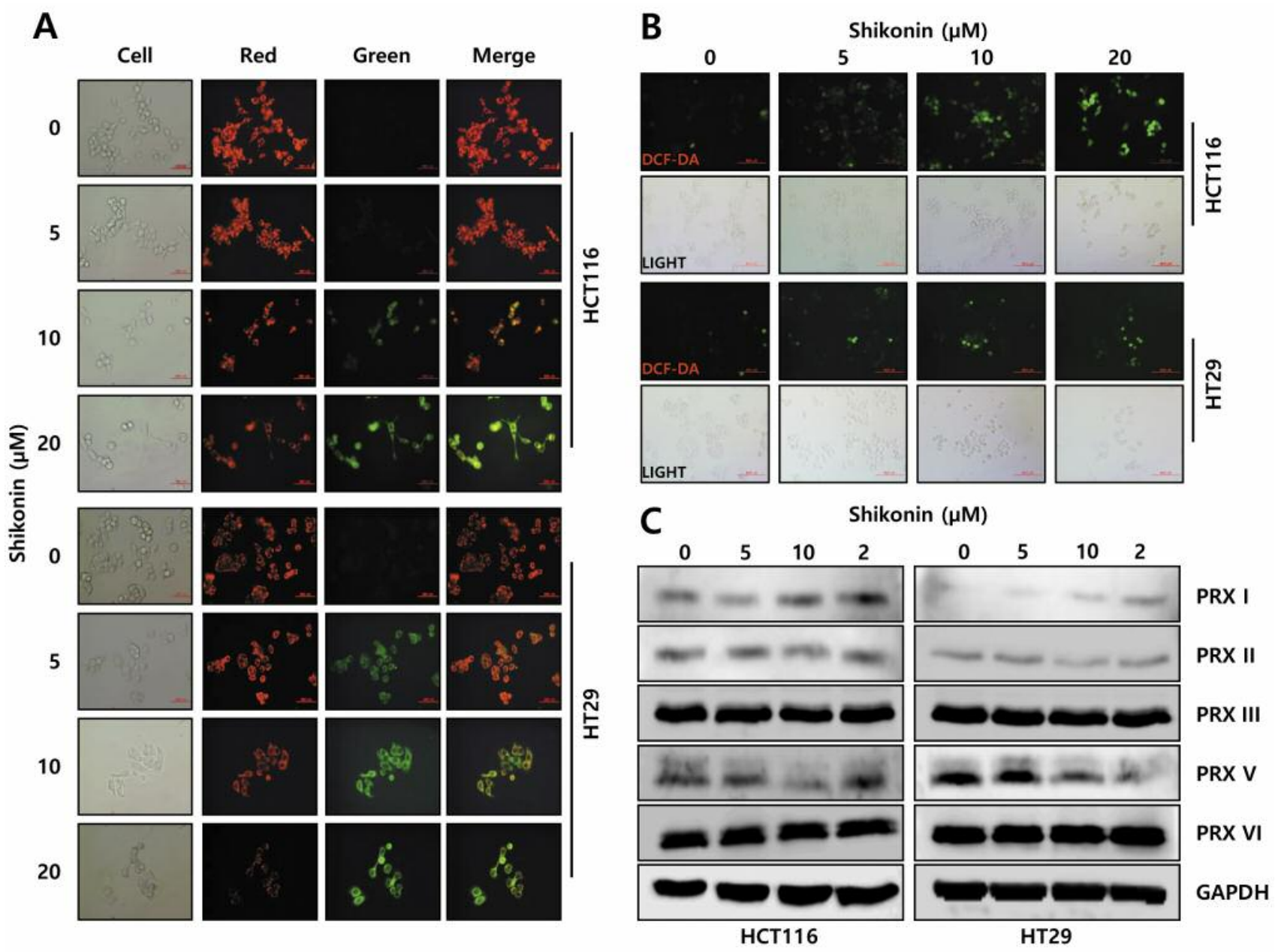

Figure 2. Role of Shikonin in oxidative status of colon cancer cells. (A) JC-1 staining of HT29 and HCT116 colon cancer cells after treatment with increasing concentrations of Shikonin to show the effects on mitochondrial reactive oxygen species (ROS) level. (B) 2',7'- Dichlorofluorescin diacetate (DCF-DA) staining of Shikonin-treated HT29 and HCT116 cells reflecting the effect of Shikonin on the ROS level of colon cancer cells. (C) Western blotting showed the dose-dependent effects of Shikonin on expression of peroxiredoxin (Prx) family members in colon cancer cells.

\section{Results}

Shikonin suppressed proliferation and malignancy of colon cancer cells. First of all, we investigated the effects of Shikonin on colon cancer cells to confirm it with previously reported effects. Shikonin has been reported to lead to ROSbased mitochondria-induced apoptosis of colon cancer cells (11). To observe the effects of Shikonin on colon cancer cells, we treated two colon cancer cell lines, HCT116 and HT29, with Shikonin and performed cell viability assay. As shown in Figure 1, HT29 and HCT116 cell viabilities were reduced by Shikonin in a dose-dependent manner. The reduction of viability was more prominent in HT29 cells compared with HCT116 cells.
From the effects of Shikonin observed in the cell viability assay, we selected four different concentrations of Shikonin $(0,5,10$ and $20 \mu \mathrm{M})$ for further experiments. As shown in Figure 1C, Shikonin also inhibited the sphere-forming ability of both colon cancer cell lines in a dose-dependent manner. Next we have performed the colony formation assay to check the effects of Shikonin on colony forming ability of colon cancer cells thereby indicating the effect on colon cancer cell proliferation. Shikonin diminished colony formation at increasing concentration (Figure 1D). Furthermore, as shown in Figure 1E and F, Shikonin treatment reduced the ability of colon cancer cells to migrate and invade. Altogether, these data confirmed that Shikonin had a dose-dependent inhibitory effect on colon cancer cell properties. 
A

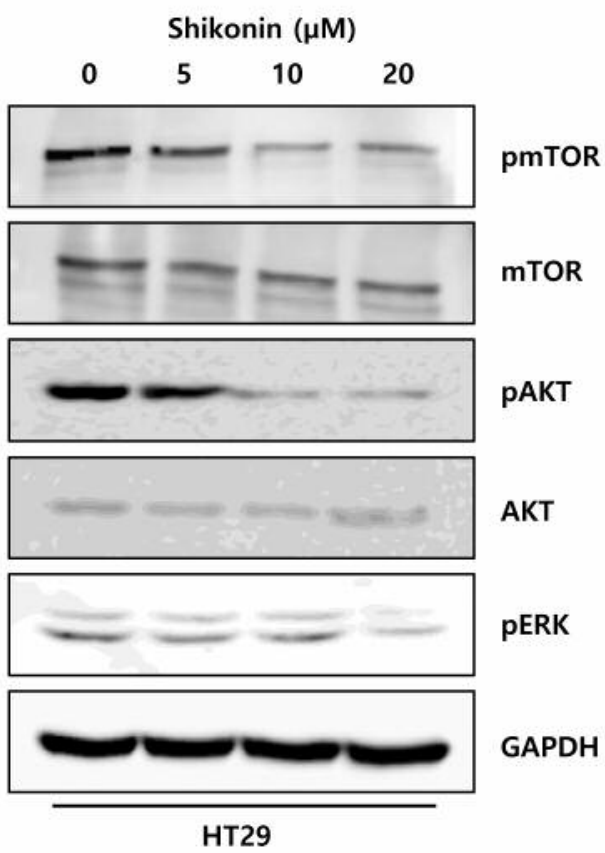

B

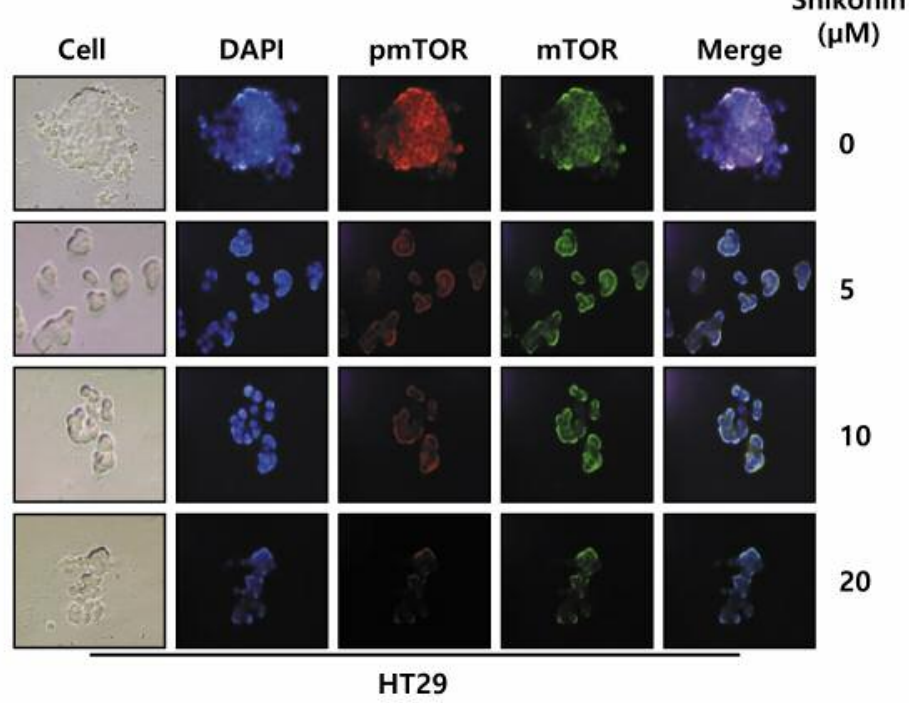

Figure 3. Shikonin mediated mammalian target of rapamycin (mTOR) signaling in HT29 colon cancer cells. (A) Western blotting to show the dosedependent effects of Shikonin on the expression of mTOR and mTOR signaling pathway-related factors. (B) Immunocytochemistry assay to confirm the effects of Shikonin on the expression of mTOR and phosphorylated (p)mTOR. AKT, Protein kinase B; ERK, extracellular signal-regulated kinase; DAPI, 4',6-diamidino-2-phenylindole; GAPDH, glyceraldehyde-3-phosphate dehydrogenase.

Shikonin affected oxidative stress and antioxidant enzymes of colon cancer cells. Next we focused on the effects of Shikonin on oxidative stress and the relationship of Shikonin with antioxidant enzymes in colon cancer cells. Shikonin has been reported to enhance cisplatin-induced colon cancer cell apoptosis as a natural inducer of ROS [8]. Moreover, Shikonin is already known for its relationship with oxidative stress of various cells in numerous ways (18-20). Similarly, we conducted JC-1 staining (Figure 2A) to check the effect of Shikonin on colon cancer cell apoptosis, mitochondrial potential, mitochondrial ROS level, and DCF-DA staining (Figure 2B) to check the effect of Shikonin on the ROS level of colon cancer cells. JC-1, a membrane-permeant dye, is widely used to monitor the healthy mitochondria mainly in apoptotic studies. One of the first stages of apoptosis is the disruption of mitochondria and a change in the oxidoreductive status of cells by alteration of the ROS level. These changes can be identified using JC-1 staining. The results showed that Shikonin induced generation of ROS in a dose-dependent manner and colon cancer cell apoptosis through induced oxidative stress.

Then the effect of Shikonin on antioxidant enzymes in colon cancer cells was investigated. The peroxiredoxin family was selected for this as peroxiredoxins are important antioxidant enzymes in most of cancer types, including colon cancer (21). As shown in Figure 2C, western blotting revealed that there were no observable effects of Shikonin treatment on PrxII, PrxIII and PrxVI. However, the expression level of PrxI was increased and that of PrxV was reduced on treatment with increasing concentration of Shikonin. These effects were more significant in HT29 cells compared with HCT116 cells. Therefore, we selected the HT29 cell line for further experiments. As we previously reported the elevated expression of PrxVcompared with other Prx family members in colon cancer cells (16), we focused on the effects of Shikonin on PrxV expression in colon cancer.

Shikonin modulates mTOR signaling in colon cancer cells. Enhanced colon cancer cell progression through induction of mTOR signaling pathway has been reported (22). Moreover, it has been reported that inhibition of mTOR signaling inhibits colon cancer cell growth (23). However, to our knowledge, the effect of Shikonin on mTOR signaling of colon cancer cells has not been studied.

Therefore, we focused on the changes of mTOR signaling induced in colon cancer cells by Shikonin treatment and further evaluated whether Shikonin inhibition of PrxV 
A

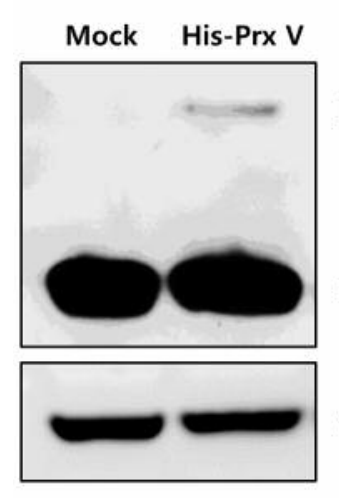

GAPDH
B

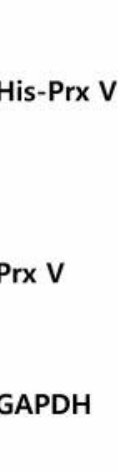

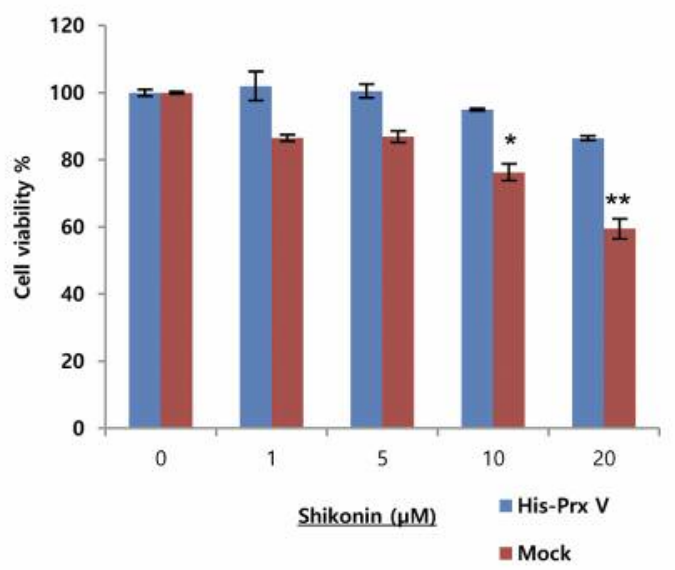

C

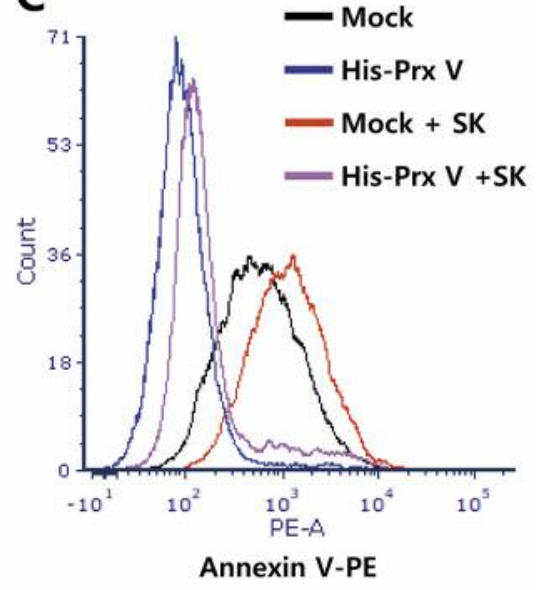

D

Shikonin $(20 \mu \mathrm{M})$

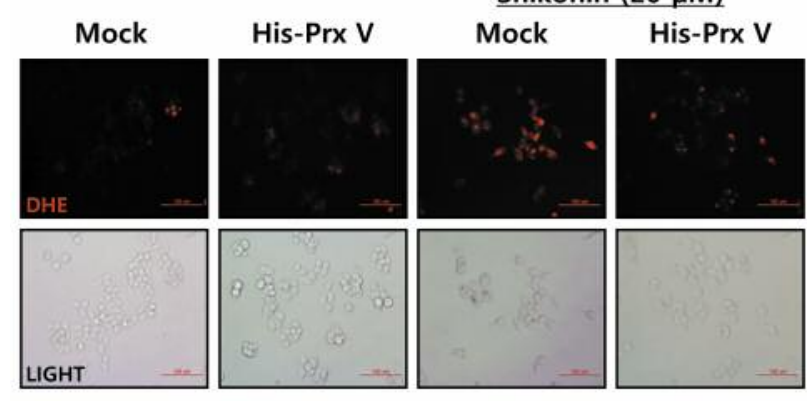

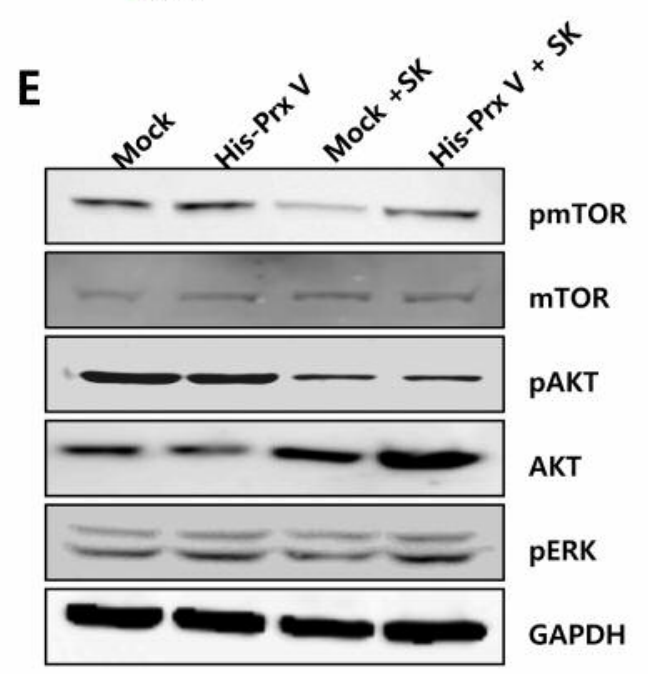

E

Figure 4. Overexpression of peroxiredoxin (progesterone receptor) V negatively regulates Shikonin-induced cell apoptosis via mammalian target of rapamycin (mTOR) signaling in colon cancer cells. (A) Western blotting to confirm the expression of Prx V in stably constructed HT29 cells. (B) MTT assay comparing the cell viability of Prx V-overexpressing colon cancer cells (His-Prx V) with control Mock cells. (C) Flow cytometric analysis of cell apoptosis. (D) Dihydroethidium (DHE) staining to check the effect of Prx V on Shikonin-induced colon cancer cell apoptosis. E: Western blotting for the effects of Prx V overexpression on mTOR signaling and related factors. AKT, Protein kinase B; ERK, extracellular signal-regulated kinase; GAPDH, glyceraldehyde-3-phosphate dehydrogenase. Differences with values of $* p<0.05, * * p<0.01$ bars represent 100 microns and $p<0.05$ values were considered statistically significant. Data is presented as the mean \pm standard error.

expression is related to mTOR signaling in colon cancer cells. Figure 3A presents western blotting results for HT29 cells treated with $0,5,10$ and $20 \mu \mathrm{M}$ of Shikonin. Even though the expression of mTOR was not significantly reduced, pmTOR was significantly down-regulated with increasing concentration of Shikonin.

Similarly, western blotting was conducted to check the expression of mTOR signaling pathway-related factors. The results showed the reduction of pAKT and pERK, while AKT expression was not changed by increasing Shikonin concentration. As shown in Figure 3C, an immunocytochemistry assay confirmed the reduction of pmTOR expression in HT29 cells with increasing Shikonin concentration. These data showed that Shikonin affects mTOR signaling in colon cancer cells in a dose-dependent manner.

Overexpression of $\operatorname{PrxV}$ reverses Shikonin-induced apoptosis and ROS level in colon cancer cells via regulation of mTOR signaling. The results in Figure $2 \mathrm{C}$ already showed that the level of PrxV was reduced with Shikonin treatment. Therefore, we examined whether this was related to Shikonin induced colon cancer cell apoptosis. For this purpose, stable HT29 colon cancer cell lines with PrxV overexpression (His- 
PrxV) and control (HT29 Mock) were established as mentioned in our previous studies $(16,17)$. Expression in cell lines was confirmed by western blotting as shown in Figure 4A.

As shown in Figure 4B, at increasing concentrations of Shikonin compared with HT29 Mock cells, viability was increased by the overexpression of PrxVas shown by MTT assay. Furthermore, as shown in Figure 4C and D, flow cytometric analysis of cell apoptosis and DHE staining showed that overexpression of $\operatorname{Prx} V$ reduced the population of apoptotic cells. As shown in Figure 4E, western blotting results showed that PrxV overexpression reversed the effect of Shikonin on the pmTOR level in HT29 cells, while no observable changes occurred in the expression of mTOR. The overexpression of PrxV did not significantly influence the expression of other factors related to mTOR signaling. Taken together, these data revealed that the HT29 cell apoptosis induced by Shikonin via regulation of the mTOR signaling pathway can be reversed by the overexpression of PrxV.

\section{Discussion}

Colon cancer has become one of the predominant cancer types in the past several decades, with higher mortality rate mainly in Western countries (24). To date, it ranks as the third most common malignancy in the world (11). Carcinogenesis of colon cancer is considered to be associated with both genetic, environmental and lifestyle factors. Even though surgical resection has remained the main treatment strategy for colon cancer, its efficiency is reduced due to the poor prognosis of colon cancer (9). Therefore, the identification of better therapeutic management strategies remains a challenge for successful colon cancer treatment.

Natural compounds extracted from various plant extracts have earned the global attention as anticancer agents (25). Shikonin, a natural naphthoquinone derivative also has been identified for its numerous anticancer effects against various cancer types (26). As a previous research group suggested, Shikonin increases colon cancer cell sensitivity to radiation therapies by inducing generation of ROS. Therefore, Shikonin is considered to be a natural inducer of ROS in cells (26). Furthermore, it has been reported that Shikonin induces colon cancer cell apoptosis through various pathways related to the endoplasmic reticulum and mitochondria (9). Shikonin was confirmed by other research groups to induce colon cancer cell apoptosis via ROS-induced mitochondrial pathways $(10,11)$. According to a previous study, Shikonin had anticancer effects on thyroid cancer cells (27). When considering the effects of Shikonin on apoptosis of melanoma cells, it was reported that this involved ROS-mediated endoplasmic reticulum stress (28). Therefore, Shikonin is widely known to induce apoptosis of various cancer cells, including colon cancer. We also confirmed these effects of Shikonin on colon cancer cells through a series of in vitro experiments (Figures 1 and 2). The underlying mechanism of Shikonininduced apoptosis in colon cancer had not been studied.

Therefore, we focused on the antioxidant enzyme system in colon cancer cells by targeting the Prx family. As we showed in our previous study, PrxV is expressed highly in colon cancer cells compared to normal colon tissues. Moreover, the expression level of PrxV is higher in colon cancer cells when compared to the expression of other Prx family members (17). In our data we found PrxV was downregulated by increasing concentrations of Shikonin. Therefore, we focused on PrxV to determine the underlying mechanism related to Shikonin-induced colon cancer cell apoptosis. Our previous study also showed that $\operatorname{PrxV}$ reduced apoptosis of colon cancer cells (16).

According to our results in this study, Shikonin induced colon cancer cell apoptosis via effects on mTOR signaling. It has been reported that the mTOR signaling pathway can induce colon cancer tumorigenesis (29). Furthermore, several studies have showed that antitumor effects can be enhanced by inhibiting mTOR signaling pathway in colon cancer cells $(30,31)$. Similarly, our results showed that Shikonin induced colon cancer cell apoptosis by down-regulating activation of mTOR signaling.

The role of PrxV was studied using HT29 His-PrxV and HT29 Mock cell lines with the treatment of Shikonin. Our results showed that the effects of Shikonin in inducing colon cancer cell apoptosis were abrogated by induced overexpression of PrxV.

We conclude that Shikonin induces colon cancer cell apoptosis by inhibiting mTOR signaling and overexpression of PrxV can abrogate the apoptosis-inducing effects of Shikonin by regulating mTOR signaling. Even though these effects of PrxV need to be studied in greater detail in future studies, our results provide new insight into understanding the underlying mechanisms to allow targeting for better clinical management of colon cancer.

\section{Conflicts of Interest}

The Authors declare no conflict of interest in regard to this study

\section{Authors' Contributions}

Nisansala Chandimali and Hu-Nan Sun designed the experiment, performed all the experiments, and Nisansala Chandimali, Hu-Nan Sun and Dong-Sun Lee wrote the article. Ling-Zu Kong, Xing Zhen and Ren Liu performed the analysis of apoptosis/ROS levels and image analysis. Taeho Kwon performed the data analysis, Dong-Sun Lee supervised the study. 


\section{Acknowledgements}

This research was supported by the Basic Science Research Program through the National Research Foundation of Korea (NRF) funded by the Ministry of Education (NRF-2016R1A6A1A03012862) and by the project for Cooperative Research Program for Agriculture Science and Technology Development (PJ01316701) Rural Development Administration, Republic of Korea.

\section{References}

1 Sousa AR, Oliveira AV, Oliveira MJ and Sarmento B: Nanotechnology-based siRNA delivery strategies for metastatic colorectal cancer therapy. Int J Pharm 568: 118530, 2019. PMID: 31323369. DOI: 10.1016/j.ijpharm.2019.118530

2 Song Y, Ye M, Zhou J, Wang ZW and Zhu X: Restoring Ecadherin expression by natural compounds for anticancer therapies in genital and urinary cancers. Mol Ther Oncolytics 14: 130-138, 2019. PMID: 31194121. DOI: 10.1016/j.omto. 2019.04.005

3 Nguyen C, Baskaran K, Pupulin A, Ruvinov I, Zaitoon O, Grewal S, Scaria B, Mehaidli A, Vegh C and Pandey S: Hibiscus flower extract selectively induces apoptosis in breast cancer cells and positively interacts with common chemotherapeutics. BMC Complement Altern Med 19(1): 98, 2019. PMID: 31060537. DOI: $10.1186 / \mathrm{s} 12906-019-2505-9$

4 Kim HJ, Hwang KE, Park DS, Oh SH, Jun HY, Yoon KH, Jeong ET, Kim HR and Kim YS: Shikonin-induced necroptosis is enhanced by the inhibition of autophagy in non-small cell lung cancer cells. J Transl Med 15(1): 123, 2017. PMID: 28569199. DOI: 10.1186/s12967-017-1223-7

5 Boulos JC, Rahama M, Hegazy MF and Efferth T: Shikonin derivatives for cancer prevention and therapy. Cancer Lett 459: 248-267, 2019. PMID: 31132429. DOI: 10.1016/j.canlet. 2019.04 .033

6 Liu T, Sun X and Cao Z: Shikonin-induced necroptosis in nasopharyngeal carcinoma cells via ROS overproduction and upregulation of RIPK1/RIPK3/MLKL expression. Onco Targets Ther 12: 2605-2614, 2019. PMID: 31118661. DOI: 10.2147/ OTT.S200740

7 Wang Z, Yin J, Li M, Shen J, Xiao Z, Zhao Y, Huang C, Zhang $\mathrm{H}$, Zhang $\mathrm{Z}$, Cho $\mathrm{CH}$ and $\mathrm{Wu} \mathrm{X}$ : Combination of shikonin with paclitaxel overcomes multidrug resistance in human ovarian carcinoma cells in a P-gp-independent manner through enhanced ROS generation. Chin Med 14: 7, 2019. PMID: 30911326. DOI: 10.1186/s13020-019-0231-3

8 He G, He G, Zhou R, Pi Z, Zhu T, Jiang L and Xie Y: Enhancement of cisplatin-induced colon cancer cells apoptosis by shikonin, a natural inducer of ROS in vitro and in vivo. Biochem Biophys Res Commun 469(4): 1075-1082, 2016. PMID: 26740178. DOI: 10.1016/j.bbrc.2015.12.100

9 Han X, Kang KA, Piao MJ, Zhen AX, Hyun YJ, Kim HM, Ryu YS and Hyun JW: Shikonin exerts cytotoxic effects in human colon cancers by inducing apoptotic cell death via the endoplasmic reticulum and mitochondria-mediated pathways. Biomol Ther (Seoul) 27(1): 41-47, 2019. PMID: 29925224. DOI: $10.4062 /$ biomolther.2018.047

10 Andujar I, Marti-Rodrigo A, Giner RM, Rios JL and Recio MC: Shikonin prevents early phase inflammation associated with azoxymethane/dextran sulfate sodium-induced colon cancer and induces apoptosis in human colon cancer cells. Planta Med 84(910): 674-683, 2018. PMID: 29642242. DOI: $10.1055 / \mathrm{a}-0599-$ 1145

11 Liang W, Cui J, Zhang K, Xi H, Cai A, Li J, Gao Y, Hu C, Liu Y, Lu Y, Wang N, Wu X, Wei B and Chen L: Shikonin induces ROS-based mitochondria-mediated apoptosis in colon cancer. Oncotarget 8(65): 109094-109106, 2017. PMID: 29312593. DOI: $10.18632 /$ oncotarget.22618

12 Huang $\mathrm{C}$ and $\mathrm{Hu} \mathrm{G}$ : Shikonin suppresses proliferation and induces apoptosis in endometrioid endometrial cancer cells via modulating miR-106b/PTEN/AKT/mTOR signaling pathway. Biosci Rep 38(2), 2018. PMID: 29449346. DOI: 10.1042/BSR20171546

13 Guo ZL, Li JZ, Ma YY, Qian D, Zhong JY, Jin MM, Huang P, Che LY, Pan B, Wang Y, Sun ZX and Liu CZ: Shikonin sensitizes A549 cells to TRAIL-induced apoptosis through the JNK, STAT3 and AKT pathways. BMC Cell Biol 19(1): 29, 2018. PMID: 30594131. DOI: 10.1186/s12860-018-0179-7

14 Zhao Q, Assimopoulou AN, Klauck SM, Damianakos H, Chinou I, Kretschmer N, Rios JL, Papageorgiou VP, Bauer R and Efferth $\mathrm{T}$ : Inhibition of c-MYC with involvement of ERK/JNK/MAPK and AKT pathways as a novel mechanism for shikonin and its derivatives in killing leukemia cells. Oncotarget 6(36): 3893438951, 2015. PMID: 26472107. DOI: 10.18632/oncotarget.5380

15 Chandimali N, Jeong DK and Kwon T: Peroxiredoxin II Regulates Cancer Stem Cells and Stemness-Associated Properties of Cancers. Cancers (Basel) 10(9): 2018. PMID: 30177619. DOI: 10.3390/cancers 10090305

16 Liu Y, Kwon T, Kim JS, Chandimali N, Jin YH, Gong YX, Xie DP, Han YH, Jin MH, Shen GN, Jeong DK, Lee DS, Cui YD and Sun HN: Peroxiredoxin V reduces beta-lapachone-induced apoptosis of colon cancer cells. Anticancer Res 39(7): 36773686, 2019. PMID: 31262894. DOI: 10.21873/anticanres.13516

17 Jin YZ, Sun HN, Liu Y, Lee DH, Kim JS, Kim SU, Jiao BY, Han YH, Jin MH, Shen GN, Lee DS, Kwon T, Xu DY and Jin YU: Peroxiredoxin V Inhibits emodin-induced gastric cancer cell apoptosis via the ROS/Bcl2 pathway. In Vivo 33(4): 1183-1192, 2019. PMID: 31280208. DOI: 10.21873/invivo.11589

18 Guo H, Sun J, Li D, Hu Y, Yu X, Hua H, Jing X, Chen F, Jia Z and $\mathrm{Xu} \mathrm{J}$ : Shikonin attenuates acetaminophen-induced acute liver injury via inhibition of oxidative stress and inflammation. Biomed Pharmacother 112: 108704, 2019. PMID: 30818140. DOI: $10.1016 /$ j.biopha.2019.108704

19 Lu B, Gong X, Wang ZQ, Ding Y, Wang C, Luo TF, Piao MH, Meng FK, Chi GF, Luo YN and Ge PF: Shikonin induces glioma cell necroptosis in vitro by ROS overproduction and promoting RIP1/RIP3 necrosome formation. Acta Pharmacol Sin 38(11): 1543-1553, 2017. PMID: 28816233. DOI: 10.1038/aps.2017.112

20 Yang JT, Li ZL, Wu JY, Lu FJ and Chen CH: An oxidative stress mechanism of shikonin in human glioma cells. PLoS One 9(4): e94180, 2014. PMID: 24714453. DOI: 10.1371/journal. pone. 0094180

21 Chandimali N, Huynh DL, Zhang JJ, Lee JC, Yu DY, Jeong DK and Kwon T: MicroRNA-122 negatively associates with peroxiredoxin-II expression in human gefitinib-resistant lung cancer stem cells. Cancer Gene Ther 26(9-10): 292-304, 2018. PMID: 30341415. DOI: 10.1038/s41417-018-0050-1

22 Ma JC, Sun XW, Su H, Chen Q, Guo TK, Li Y, Chen XC, Guo J, Gong ZQ, Zhao XD and Qi JB: Fibroblast-derived CXCL12/SDF-1alpha promotes CXCL6 secretion and co- 
operatively enhances metastatic potential through the $\mathrm{PI} 3 \mathrm{~K} / \mathrm{Akt} / \mathrm{mTOR}$ pathway in colon cancer. World J Gastroenterol 23(28): 5167-5178, 2017. PMID: 28811711. DOI: 10.3748/wjg.v23.i28.5167

23 Ponnurangam S, Standing D, Rangarajan P and Subramaniam D: Tandutinib inhibits the Akt/mTOR signaling pathway to inhibit colon cancer growth. Mol Cancer Ther 12(5): 598-609, 2013. PMID: 23427297. DOI: 10.1158/1535-7163.MCT-12-0907

24 Kuipers EJ, Grady WM, Lieberman D, Seufferlein T, Sung JJ, Boelens PG, Van De Velde CJ and Watanabe T: Colorectal cancer. Nat Rev Dis Primers 1: 15065, 2015. PMID: 27189416. DOI: $10.1038 / \mathrm{nrdp} .2015 .65$

$25 \mathrm{Je}$ HD, Kim HD and La HO: The inhibitory effect of shikonin on the agonist-induced regulation of vascular contractility. Biomol Ther (Seoul) 23(3): 233-237, 2015. PMID: 25995821. DOI: $10.4062 /$ biomolther.2014.148

26 Kwak SY, Jeong YK, Kim BY, Lee JY, Ahn HJ, Jeong JH, Kim MS, Kim J and Han YH: beta,beta-Dimethylacrylshikonin sensitizes human colon cancer cells to ionizing radiation through the upregulation of reactive oxygen species. Oncol Lett 7(6): 1812-1818, 2014. PMID: 24932238. DOI: 10.3892/ol.2014.2018

27 Hasenoehrl C, Schwach G, Ghaffari-Tabrizi-Wizsy N, Fuchs R, Kretschmer N, Bauer R and Pfragner R: Anti-tumor effects of shikonin derivatives on human medullary thyroid carcinoma cells. Endocr Connect 6(2): 53-62, 2017. PMID: 28069896. DOI: $10.1530 / \mathrm{EC}-16-0105$
28 Liu Y, Kang X, Niu G, He S, Zhang T, Bai Y, Li Y, Hao H, Chen $\mathrm{C}$, Shou $\mathrm{Z}$ and Li B: Shikonin induces apoptosis and prosurvival autophagy in human melanoma A375 cells via ROS-mediated ER stress and p38 pathways. Artif Cells Nanomed Biotechnol 47(1): 626-635, 2019. PMID: 30873870. DOI: 10.1080/21691401. 2019.1575229

29 Wei Q, Zhang B, Li P, Wen X and Yang J: Maslinic Acid Inhibits Colon Tumorigenesis by the AMPK-mTOR Signaling Pathway. J Agric Food Chem 67(15): 4259-4272, 2019. PMID: 30913881. DOI: $10.1021 /$ acs.jafc. 9 b00170

30 Chen Y, Lee CH, Tseng BY, Tsai YH, Tsai HW, Yao CL and Tseng SH: AZD8055 exerts antitumor effects on colon cancer cells by inhibiting mTOR and cell-cycle progression. Anticancer Res 38(3): 1445-1454, 2018. PMID: 29491070. DOI: 10.21873/ anticanres.12369

31 Wu SY, Huang YJ, Tzeng YM, Huang CF, Hsiao M, Wu ATH and Huang TH: Destruxin B suppresses drug-resistant colon tumorigenesis and stemness is associated with the upregulation of mir-214 and downregulation of mTOR/beta-catenin pathway. Cancers (Basel) 10(10), 2018. PMID: 30257507. DOI: $10.3390 /$ cancers 10100353

Received September 14, 2019

Revised September 27, 2019 Accepted October 7, 2019 